less been the product of much work on the part of their authors and which are rendered almost useless by the fact that the colors are designated by fanciful names peculiar to the output of one firm. In one case in mind, the firm, whose colors are treated at some length, probably does not make one gram of dye. The dyes are purchased and, after being "reduced" and suitably compounded, are sold under coined names. The confusion of well-known trade names as given in Allen or Schultz and Julius is bad enough. Besides this, it is certainly a fact that many of the colors sold by the large manufacturers under well-known names are mixtures. Some days ago the writer received a line of eosine samples from one of the largest manufacturers. Each had a definite and familiar name, but upon further inquiry it was admitted that three out of five were mixtures. In view of these facts it would seem very necessary that more attention should be given to the identity and purity of colors used in constructing tables for their detection and identification.

NEW YORK LABORATORY, BUREAU OF CHEMISTRY,

U. S. DEPARTMENT OF AGRICULTURE.

\title{
COMPOSITION OF KNOWN SAMPLES OF PAPRIKA.
}

By $R$ E. DOOIITTLE AND A. W. OGDEN.

Received July $14,1908$.

Because of cheaper labor in the countries of production, practically all paprika reaches the markets of this country in a ground state, and like other ground spices it is more or less subject to sophistication. Being valued for its sweet flavor and high color the temptation to add artificial coloring matter to inferior grades to make them appear of a higher grade is very great and is quite commonly practiced. The color of the inferior grades is also brought up by grinding the pods with olive oil. The various grades of paprika are produced by selection of pods and the removal of the stems and seeds therefrom, for it is in the seeds and placentae to which the seeds are attached that are located the sharp and pungent properties of the fruit, which are not desired in the finished product. The finer grades of paprika are, therefore, produced by grinding the selected, well ripened and highly colored pods from which the stems and more or less of the seeds are removed. The lower grades are from the lower grade pods, from which the seeds or even the stems are not removed or to which the superfluous seeds and stems of the finer grades are sometimes added. By these various sortings and mixtures, products of all grades and prices are produced, even to the so-called "Mercantile" grade, which is composed entirely of ground stems and other refuse.

In order that we might have at hand reliable data by which to judge the character of the importations of the ground paprika presented for entry to this country we secured direct from the producers large samples 
of the whole paprika pods. From these we first selected a representative sub-sample and determined the proportion of stems, shell, and seeds in same. Other samples were then taken and separated into four classes, namely, first, a representative sample of the whole pods as received; second, shells, the stems, seeds, and placentae being removed; third, the seeds and placentae; fourth, stems. Each of these classes were ground and examined separately.

Methods of Analysis.--The official methods for the analysis of spices (L.S. Dept. of Agriculture, Bureau of Chemistry, Bulletin I07, page 162) were followed for the determination of ash, ether extract, crude fiber, reducing sugars and nitrogen.

Alkalinity of ash soluble in water was determined by titrating the filtrate obtained from ash insoluble in water against O.I $N$ hydrochloric acid, using methyl orange as indicator.

Alkalinity of total ash was determined by adding an excess of tenthnormal hydrochloric acid to ash from 2 grams of material, boiling to remove carbon dioxide, and titrating back with tenth-normal sodium hydroxide, using methyl orange as indicator.

The iodine absorption number of the ether extract was determined in the following manner, using the Hanus iodine solution (U.S. Dept. of Agriculture, Bureau of Chemistry, Bulletin 107, page 136). Two grams of the ground paprika were extracted on a dried filter paper with anhydrous ether, collecting the washings in a tared ground glass stoppered flask. The ether was removed by distillation at low temperature and the extract dried for thirty minutes in the steam oven. The flask was then allowed to cool in the air for thirty minutes and weighed, and the drying, cooling, and weighing were repeated until the loss in weight did not exceed I mg. If care was taken to remove all ether fumes by gently twirling the flasks in a horizontal position before placing them in the oven and to lay the flasks on the side while drying, thirty minutes' drying in the steam oven was found sufficient. The extractive matter was then dissolved in the flask by the addition of ro cc. of chloroform or carbon tetrachloride, and shaking; $30 \mathrm{cc}$. of the Hanus iodine solution were then added and the flask allowed to stand with occasional shaking for thirty minutes; ro cc. of a $I_{5}$ per cent. potassium ioclide solution were added and the contents of the flask well shaken; then $135 \mathrm{cc}$. of distilled water and the whole was titrated against $0.1 \mathrm{~N}$ thiosulphate solution in the usual manner. Blanks were run under exactly the same conditions. It was found that when the manipulation was carried out carefully according to the above method very concordant results were obtained. It is to be noted, however, that anhydrous ether must be used for the extraction of the powdered sample.

Each of the samples of the whole pods and the powdered shells were submitted to the double dyeing method of Sostegni Carpentieri for the 


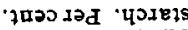

s E p

แoเรณว

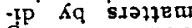

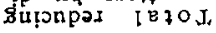

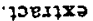

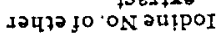

3400 มวd

$92.9 \times \mathbf{N}$ u!zjodd

IPd $428077 ! \mathrm{N}$

ถูก

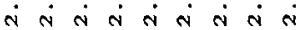

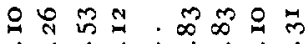

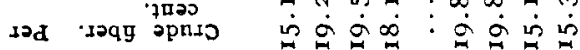

. 2420

Iวद

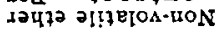

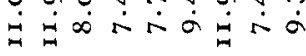

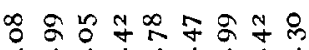

$\stackrel{n}{0}: \stackrel{n}{0}$

is $\rightarrow m$ 它官息

ஸै $\dot{j} \dot{0} \dot{n}$

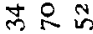
तं वं

ㄱำ

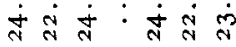

N+ $00000 \mathrm{n}$

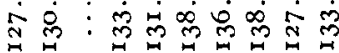

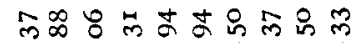

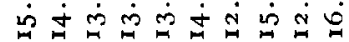

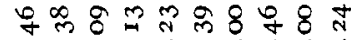

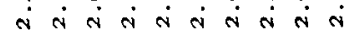

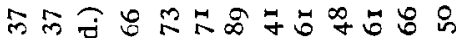
: ம் की: की

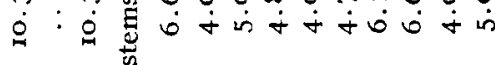

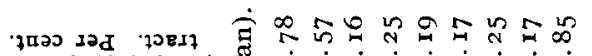

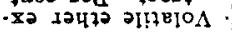

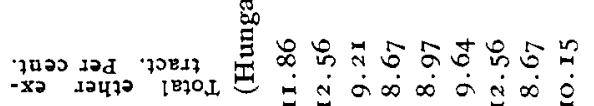

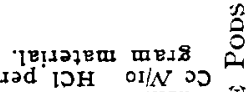

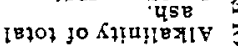

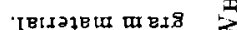
Iad IDH oIN

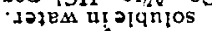

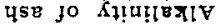

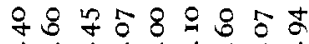

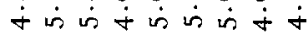

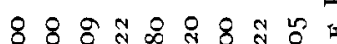
$\dot{\Delta} \dot{\infty} \dot{0} \dot{0} \dot{\sim} \dot{0} \dot{0}$ 西

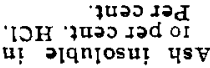

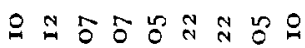
$\begin{array}{llllllll}0 & 0 & 0 & 0 & 0 & 0 & 0 & 0\end{array}$

ๆ पा ग โq

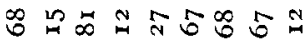

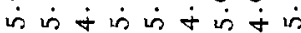

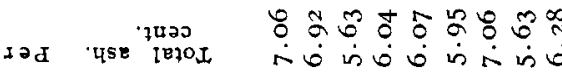

4

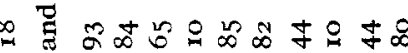

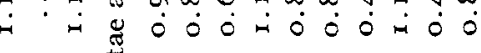

的 8 造

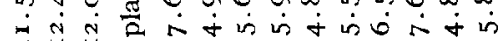

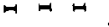

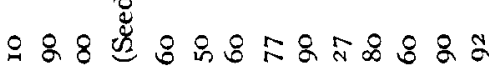

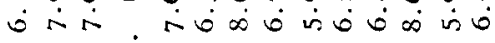

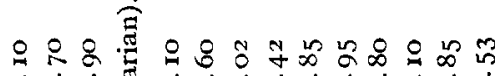

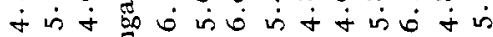
两自

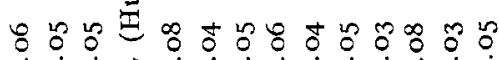

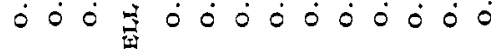
오ㅇㅛㅛ $\dot{f} \dot{\text { in }}$

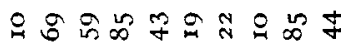

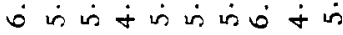
in $\dot{0}$

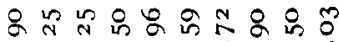
$\dot{0} \dot{0} \dot{0}$ in $\dot{\text { ns }}$ is $\dot{0}$ 的 $\dot{0}$

7uas rod .

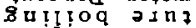

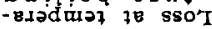

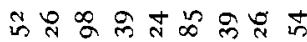
$\infty \therefore \dot{0} \dot{0} \dot{0} \dot{0} \dot{0}$

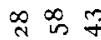
$\infty \infty \infty$

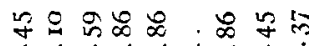
a $\dot{0} \dot{0} \dot{0} \dot{0} \dot{0} \dot{0}$ 


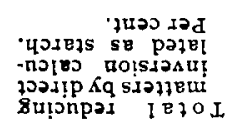

. \&. \& \& 8 $\dot{a}: \dot{\sigma} \dot{\sigma}$

8utonpas le70I

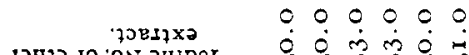

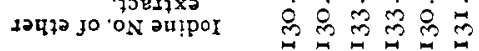

7horad

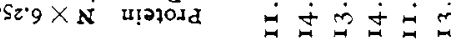

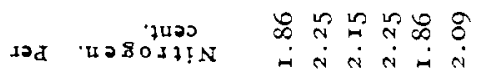

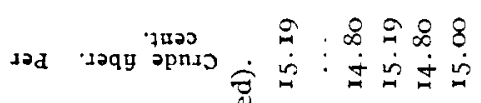

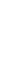

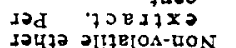

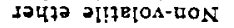 \\ juכ2 I09 joest

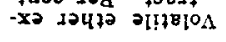

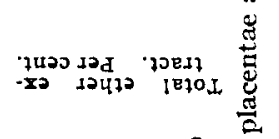

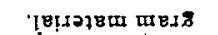

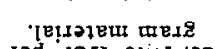

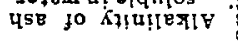

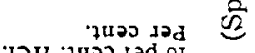

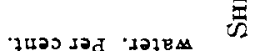

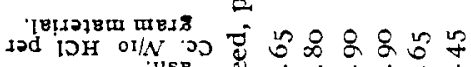
[ह107 50 Ki!

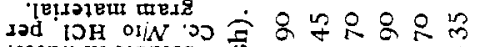

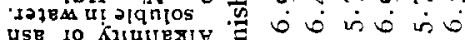

त 5 용=

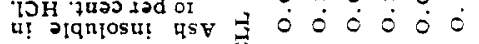

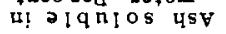

$\infty \stackrel{\infty}{0}: 08$ b i $\dot{0}$ is is

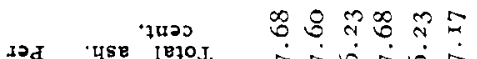

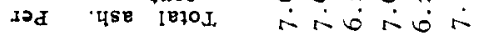

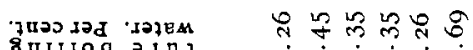

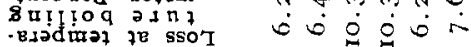

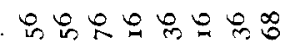

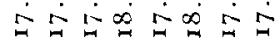

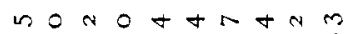
$\dot{m} \dot{m} \dot{m} \dot{m} \dot{m} \dot{m} \dot{m} \dot{m} \ddot{m}$

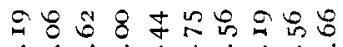

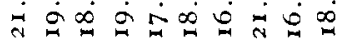

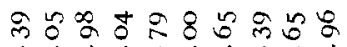
$\dot{\Rightarrow} \dot{\sim} \dot{N} \dot{N} \dot{\sim} \dot{N} \dot{m} \dot{N} \dot{N}$

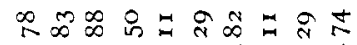

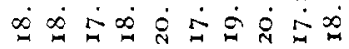

유ㅇㅠㅀㅠ तें

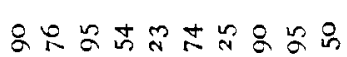

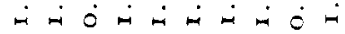
88 융ำ요용

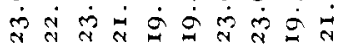

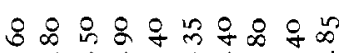

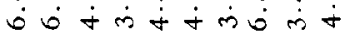

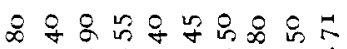

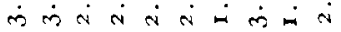

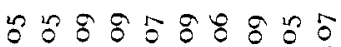

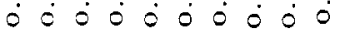
$\because N \mathbb{N}$

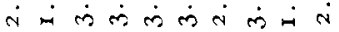

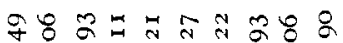

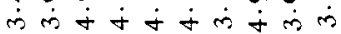

용 8 웅 is is is 0 o 0 is 


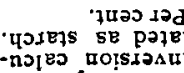

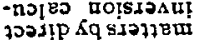

8utinper I

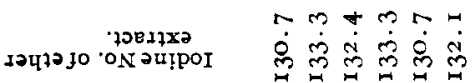

.

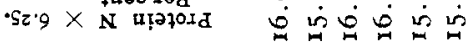

Iจd

in $\infty 8 g 0 \infty$ i तो ले

\&

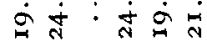

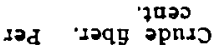

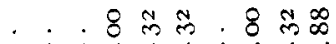

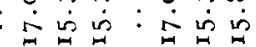

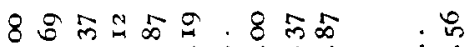

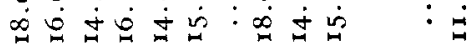

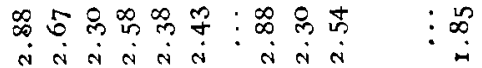

Iง

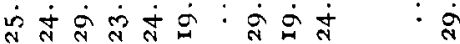

$\operatorname{rad} .708 \pi, 7202$

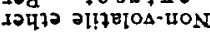

\&: 용ㅇㅇ $\dot{0} \dot{\theta} \underset{\sim}{\infty} \dot{\sigma}$

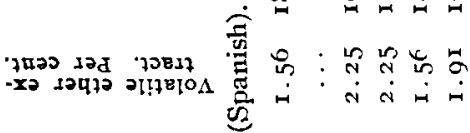

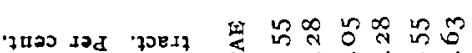

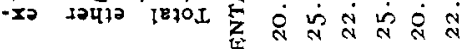
ก Jวव

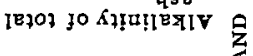

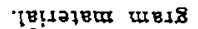

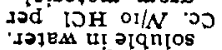
पse jo Kitu!lextv

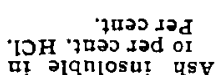
$8 \otimes \& 8 \%$

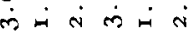

융ㅇㅇㅇㅛ $\dot{j} \dot{\dot{\theta}} \dot{+} \dot{m} \dot{+}$

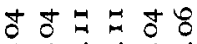

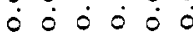

यू मून

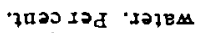
น! गाดा

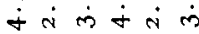

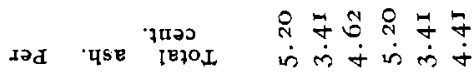


detection of synthetic coloring matters (U.S. Dept. of A griculture, Bureau of Chemistry, Bulletin I07, page I36) and none were found that gave any color to the wool from the second bath. It was found preferable, however, to thoroughly wash the wool with a strong soap solution, when taken from the first bath, in order to remove all oil and resin. Samples colored with the ordinary oil-soluble synthetic dyes were readily detected by this test.

The results of the examinations made are tabulated below. It will be noted that paprika produced from the shells alone contains not to exceed 7.50 per cent. of ash, and not to exceed 8.00 per cent. total ether extract, having an iodine number of not less than 127 , while paprika made from the whole pods contains not to exceed 7.50 per cent. ash, and not to exceed I 3.00 per cent. of total ether extract, having an iodine number of not less than i 30 .

Percentage of Shelit, Seeds and Stems in Whole Pods.

$\begin{array}{clccc}\text { Lab No. } & \text { Variety. } & \text { Shell. } & \text { Seeds and placentae. } & \text { Stems, } \\ 20 \ldots \ldots & \text { Hungarian } & 64.2 & 26.4 & 9.4 \\ 28 \ldots \ldots & \text { Hungarian } & 64.3 & 27.4 & 8.3 \\ 41 \ldots \ldots & \text { Hungarian } & 56.6 & 40.4 & 3.0 \\ 33 \ldots \ldots & \text { Spanish } & 55.0 & 36.2 & 8.8\end{array}$

BUREAU OF CHEMISTRY,

U. S. DEPARTMENT OF AGRICULTURE,

WASHINGTON, D. C.

\section{THE NATURE OF THE VOLATILE MATTER OF COAL AS EVOLVED UNDER DIFFERENT CONDITIONS. ${ }^{1}$}

By Horace C. PORTER and F. K. OVITz.

Received June $30,1908$.

The experiments described below have been a part of the fuel investigations conducted during the year 1907-1908 by the Technologic Branch, $U$. S. Geological Survey. The investigation is still in progress along the same and similar lines.

It is a familiar fact to retort coke oven and gas works operators that the volatile products of coal are largely affected both in quantity and character by the conditions of temperature and rapidity of the rise of temperature in the coal, and by the conditions to which the products are subjected after leaving the coal. The laboratory determination of volatile matter serves almost universally as a more or less valuable indication of the coal's adaptability to industrial uses either for combustion, destructive distillation or gasification. The method for this determination is, however, an arbitrary one and does not duplicate closely that of any industrial operation, nor is the character of the volatile matter produced by the laboratory method known with any degree of certainty.

${ }^{1}$ Presented with the permission of the Director, U. S. Geological Survey. 